\title{
Electrochemical Fabrication of Porous Silicon Structures for Solar Cells
}

\author{
R. S. Dubey \\ Department of Nanotechnology, \\ Advanced Research Laboratory for Nanomaterials and Devices, \\ Swarnandhra College of Engineering and Technology, Seetharampuram, Narsapur (A.P.), India \\ *Corresponding Author: rag_pcw@yahoo.co.in
}

Copyright (C) 2013 Horizon Research Publishing All rights reserved.

\begin{abstract}
Porous Silicon (PS) has found its broad application for optoelectronics devices specially solar cells applications due to its efficient antireflection coating. This material has advantages such as broaden band gap, wide absorption spectrum and high optical transmission range from $700-1000 \mathrm{~nm}$. In this paper, the experimental study of electrochemically prepared porous silicon structures is presented. With an increase in etching time, the thickness and refractive index of porous silicon were found to be increased and decreased respectively. The XRD measurement has confirmed the crystallinity nature of porous silicon as that of bulk silicon. A broad peak of red emission has also confirmed by photoluminescence measurement and it has been attributed to $\mathrm{SiHx}$ groups which are confirmed by FTIR spectra. Pyramid like surface over the entire surface is confirmed by AFM measurement which is responsible for the reduction of reflection of photons and hence can enhance the conversion efficiency of solar cells.
\end{abstract}

Keywords Porous Silicon, PL, AFM, Reflection, Antireflection Coating, Solar Cells

\section{Introduction}

An increasing interest has been shown towards antireflection coating layer made from porous silicon due to its low fabrication cost. Porous silicon has been attracted great attention due to its room temperature photoluminescence in the visible light range after the pioneer work of Canham [1]. In order to reduce the cost of solar panels, there is a need of enhance the conversion efficiency of solar cells so that photons falling onto solar panels can generate electron and hole pairs which can be collected at the two points. However, the semiconductor materials used for solar cells are sensitive to a part of the solar radiation spectrum. In this view, porous silicon is a promising one as it has the capability of a wide absorption bands and high transmission from 700 to $1000 \mathrm{~nm}$ [2]. For solar cells, porous silicon layer acts as an antireflection coating while graded layer with varying expanded band gap offer increased absorption in visible spectrum region. Porous silicon shows different features in comparison to the bulk silicon such as shifting of fundamental absorption edge into the short wavelength and photoluminescence in the visible region of the spectrum [3]. Porous silicon is a very promising material due to its excellent properties and compatibility with silicon based microelectronics with reduced fabrication cost. The most important advantages of using porous silicon in solar cells are its textured nature surface which traps light and reduces the reflectance losses, tuning of band gap for optimum sunlight absorption, conversion into direct band gap semiconductor with large quantum efficiency. Electrochemical is an easy method for porous silicon formation which employs a platinum cathode and silicon wafer anode immersed in hydrofluoric acid (HF) electrolyte with constant current source, which is usually implemented to ensure steady tip concentration of HF resulting in a more homogenous porosity layer. Porous silicon structures has good mechanical robustness, chemical stability and compatibility with existing silicon technology therefore has a wide area of potential applications such as waveguides, 1D photonic crystals, chemical sensors, biological sensors, photovoltaic devices etc.[4-10].

In this paper, the electrochemically anodized porous silicon structures have been characterized for various measurements. The experimental procedure of electrochemical anodization has been presented in section 2 while analysis of characterized results is summarized in section 3 and finally, section fourth concludes the paper.

\section{Experimental Procedure}

For electrochemical anodization, boron doped P-type Silicon substrate of $\langle 100>$ orientation was used. Before electrochemical etching of silicon, silicon substrates were rinsed in de-ionized water after heating in trichloroethylene for 5 minutes. In second step, silicon substrates were again 
heated in acetone for 5 minutes, and rinsed in de-ionized water. In third step, the silicon substrates were again heated in methanol for 5 minutes, and then rinsed in de-ionized water. Finally, the cleaned silicon substrates were dried in the presence of nitrogen and used for anodization. For anodization, CH1100A (USA) is used as constant DC current source with electrolyte solution containing 1:1:2 ratio of HF(48\%): DI water : Ethanol respectively. In case of use of purely aqueous HF solutions, for the formation of PS formation; hydrogen bubbles stick to the surface and induce lateral and in-depth inhomogeneity. However, the addition of ethanol to HF eliminates hydrogen and ensures complete infiltration of HF solution within the pores which further improves the uniform distribution of porosity and thickness. The electrochemical cell used has two electrode configurations with a platinum electrode as cathode and silicon wafer as anode. As the starting material was of P-type Silicon substrate hence, anodization was carried out at room temperature in the dark. Various samples were fabricated by changing the anodization time, current density and HF concentration. The prepared samples were characterized by ellipsometry, Photoluminescense, Fourier transform infrared transform spectroscopy (FTIR), X-ray diffraction (XRD) and atomic force microscopy (AFM).

\section{Results \& Discussion}

The properties of fabricated porous structures can be tuned with respect to process parameters such as concentration of electrolyte solution, current density and etching time. The two most important characteristic of a porous silicon layer are its refractive index and thickness which are mainly depending on anodization process: i.e. current density and anodization time. Figure 1 shows the variation of refractive index with respect to anodization time for two distinct current densities.

As the etching time increased, the refractive index of porous silicon layer is deceased. However, reduction in refractive index can be observed for the sample prepared at current density $\mathrm{J}=50 \mathrm{~mA} / \mathrm{cm}^{2}$. This reduction in refractive index was due to an increased of porosity.

Similarly, figure 2 shows the variation in the thickness of porous silicon layers as function of etching time prepared at $\mathrm{J} 1=30$ and $\mathrm{J} 2=50 \mathrm{~mA} / \mathrm{cm}^{2}$ respectively.

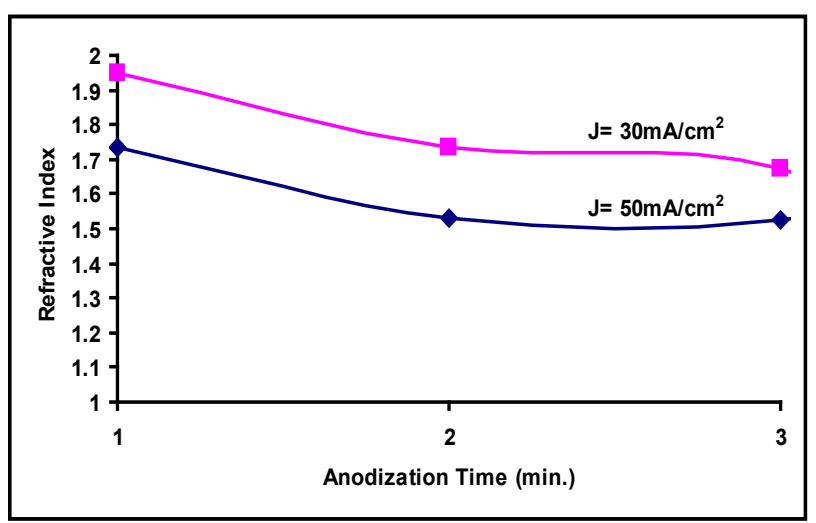

Figure 1. Refractive index of porous silicon with anodization time.

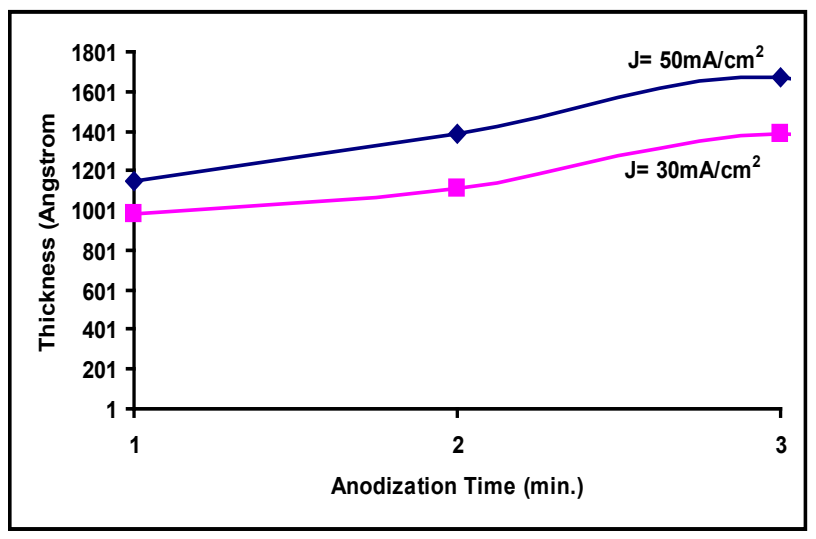

Figure 2. Porous Silicon thickness as a function of anodization time.

An increment of porous layer thickness can be observed with increased etching time. With figure 1 and 2, we have concluded that the required refractive index and thickness profile can be achieved by controlling the anodization time and applied current density. Table (I) shows the measured value of refractive index and thickness of porous silicon layers prepared at 30 and $50 \mathrm{~mA} / \mathrm{cm}^{2}$ current density respectively.

Table 1

\begin{tabular}{|c|c|c|c|c|c|}
\hline Sample & $\begin{array}{c}\text { Electrolyte } \\
\text { (HF:DI: } \\
\left.\mathrm{C}_{2} \mathrm{H}_{5} \mathrm{OH}\right)\end{array}$ & Current Density & Etching time & $\begin{array}{c}\text { Thickness } \\
\left(\mathrm{A}^{\mathrm{o}}\right)\end{array}$ & Refractive Index \\
\hline S1 & $1: 1: 2$ & $30 m A$ & $1 \mathrm{~min}$. & 981 & 1.95 \\
\hline$S 2$ & $1: 1: 2$ & $30 m A$ & 2 mins. & 1112.3 & 1.735 \\
\hline$S 3$ & $1: 1: 2$ & $30 m A$ & 3 mins. & 1390.26 & 1.676 \\
\hline S4 & $1: 1: 2$ & $30 m A$ & 4 mins. & 1122.3 & 1.2 \\
\hline S5 & $1: 1: 2$ & $50 m A$ & $1 \mathrm{~min}$. & 1150 & 1.735 \\
\hline S6 & $1: 1: 2$ & $50 m A$ & 2 mins. & 1392 & 1.533 \\
\hline$S 7$ & $1: 1: 2$ & $50 m A$ & 3 mins. & 1670 & 1.523 \\
\hline$S 8$ & $1: 1: 2$ & $50 m A$ & 4 mins. & 1176 & 1.75 \\
\hline
\end{tabular}




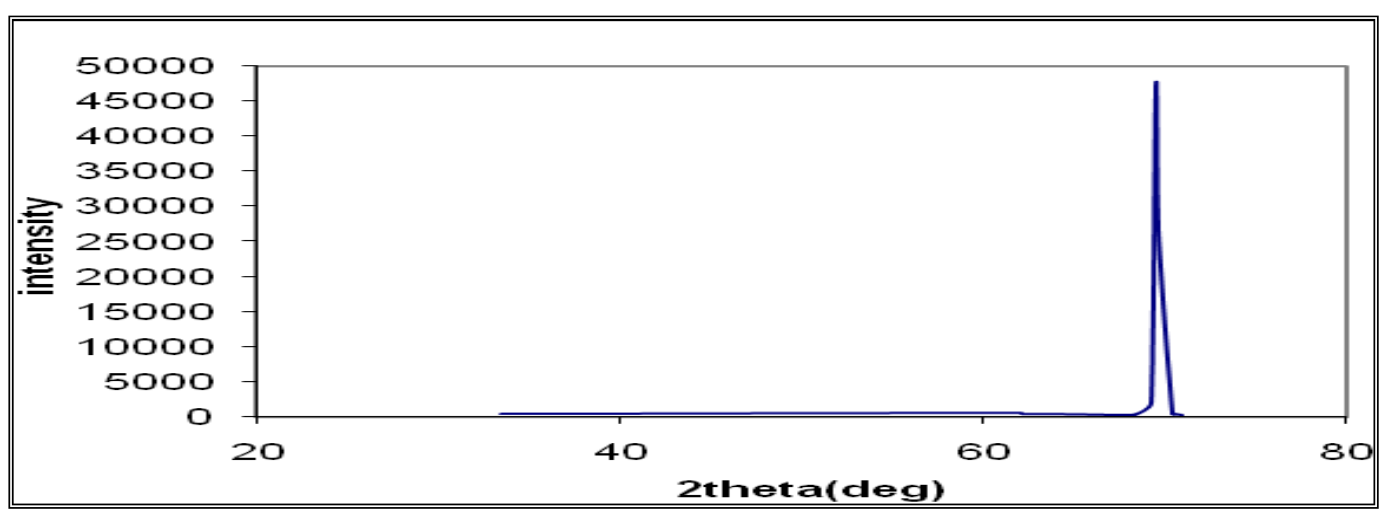

Figure 3. XRD spectra of porous silicon prepared $\mathrm{J}=30 \mathrm{~mA} / \mathrm{cm}^{2}$.

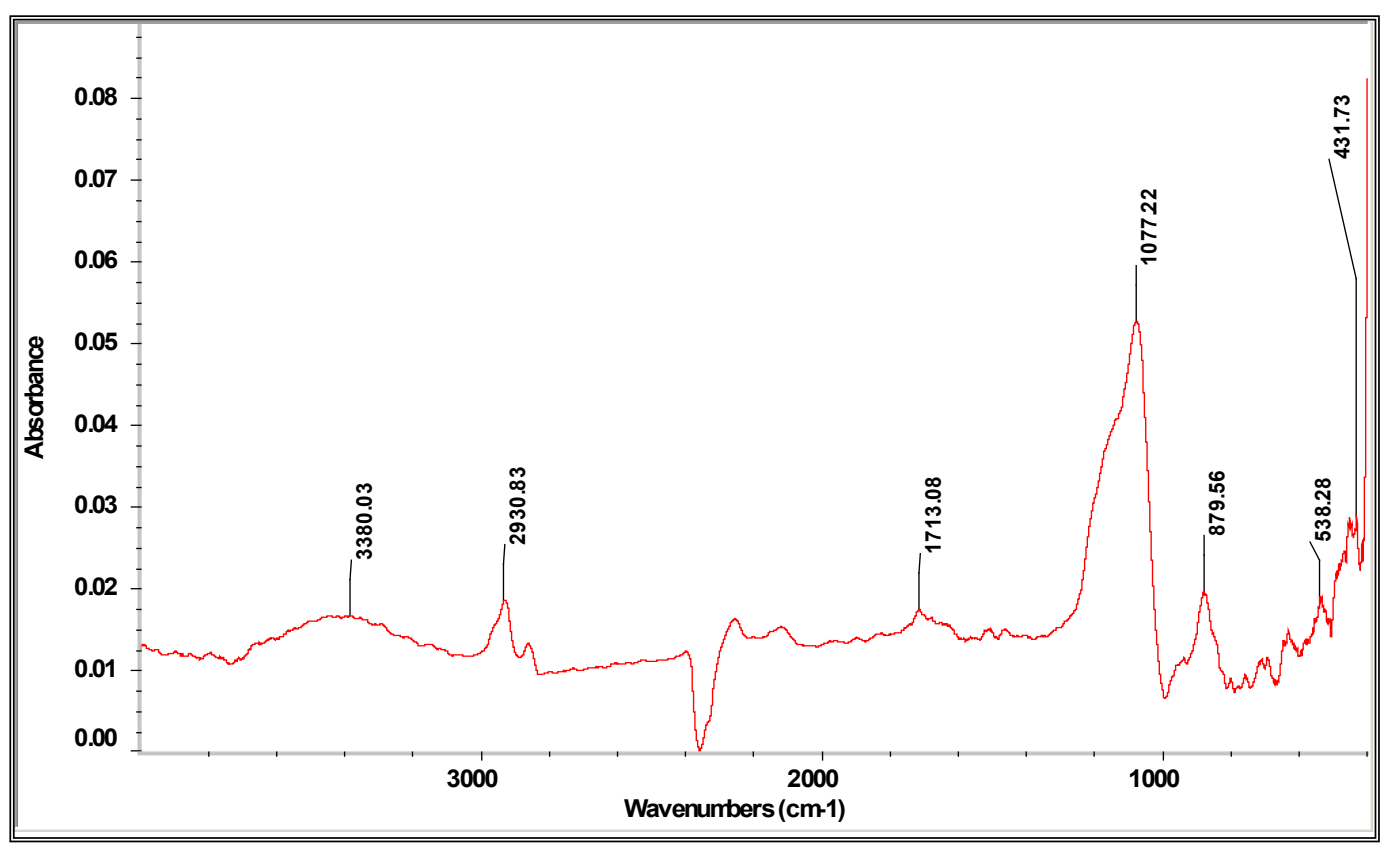

Figure 4. FTIR spectra of porous silicon prepared at $\mathrm{J}=30 \mathrm{~mA} / \mathrm{cm}^{2}$.

Table II

\begin{tabular}{|c|c|c|}
\hline Absorption band(cm-1) & Mode & Vibrational mode \\
\hline 431 & $\mathrm{Si}-0-\mathrm{Si}$ & Bending \\
\hline 538 & $\mathrm{Si}-\mathrm{Si}$ & Stretching \\
\hline 879 & $\mathrm{O}_{3} \mathrm{Si}-\mathrm{H}$ & Bending \\
\hline 1077 & $\mathrm{Si}-\mathrm{OH}$ & Stretching \\
\hline 1713 & $\mathrm{C}=\mathrm{O}$ & Carbon oxygen bond \\
\hline 2930 & $\mathrm{CH}_{2}$ & Asymmetric stretching \\
\hline 3380 & $\mathrm{OH}$ & Stretching, water vapor \\
\hline
\end{tabular}

To confirm the crystallographic perfection of the starting material with resulting porous structure, XRD measurement has done and shown in figure 3. In XRD spectra, a sharp peak can be observed at $2 \theta=69^{\circ}$ which indicates the single nature of silicon of prepared sample $\mathrm{J}=30 \mathrm{~mA} / \mathrm{cm}^{2}$.

Figure 4 shows the FTIR spectra of porous structure prepared at $\mathrm{J}=30 \mathrm{~mA} / \mathrm{cm}^{2}$.

This spectra is measured by Nicolet 380 FTIR
Spectrometer in the range of $400 \mathrm{~cm}^{-1}$ to $4000 \mathrm{~cm}^{-1}$ wave numbers which contains the fundamental vibration frequency of the main bonding groups of almost any material including the silica-based ones. The important observed IR peaks are listed in table (II).

These peak positions are compared with others reported work and have found good matching. For the application of solar cells, top layer is required to be low reflective surface. 


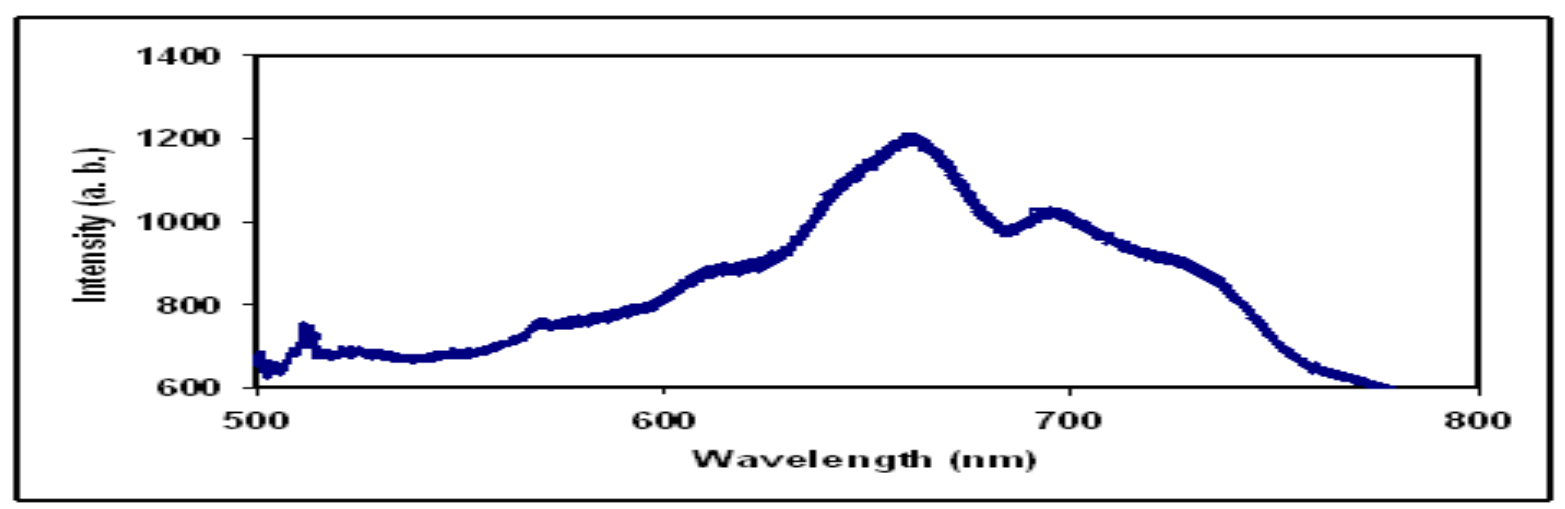

Figure 5. Photoluminescence of porous silicon.

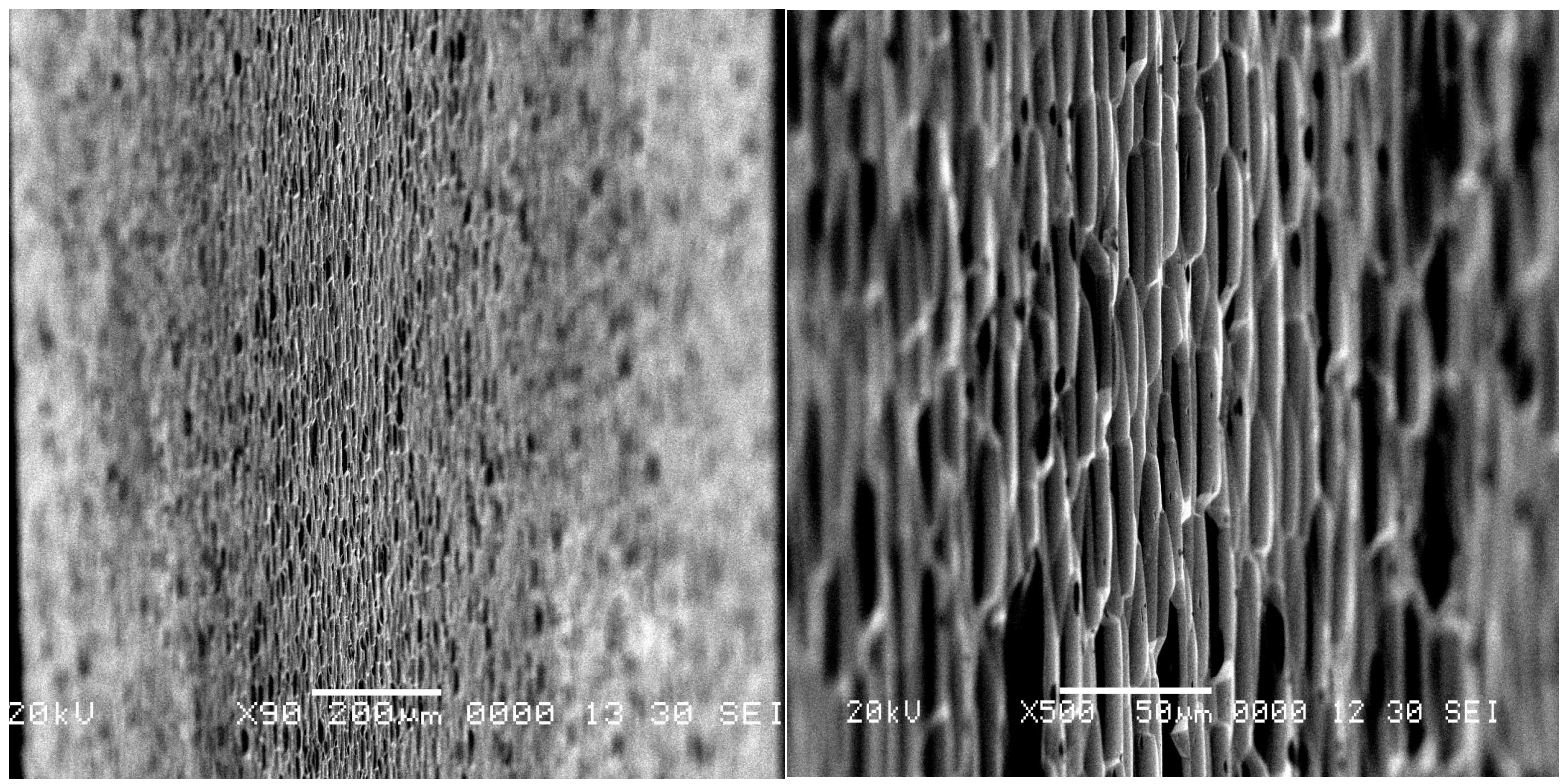

Figure 6. Surface morphology of prepared porous silicon..

Figure 5 shows the photoluminescence (PL) of sample prepared at current density $50 \mathrm{~mA} / \mathrm{cm}^{2}$ under 3 mins. etching time. A peak of red band emission can be observed at $662 \mathrm{~nm}$.

It is noted that the surface hydrides on the surface of porous silicon are responsible for the 'red' band PL and it has been attributed to SiHx groups which is confirmed by FTIR spectra.

From 6 shows the SEM of prepared porous structure at $50 \mathrm{~mA} / \mathrm{cm}^{2}$ current density under 3 mins. etching time. The porous formation can be confirmed (left side) however the existence of silicon nanostructure is clearly visible (right side).

Figure 7 shows the three dimensional topographic image of porous silicon with the pyramid like shape distributed over the entire surface. The pyramidal shape point towards an increase in the surface roughness resulted due to anodization.

High degree of roughness of the porous silicon surface implies the possibility of its application as antireflection coating as textured surface reduces the light reflection. Further, scattering in porous silicon is possible due to the roughness in relation to the thickness of the porous layer [16].

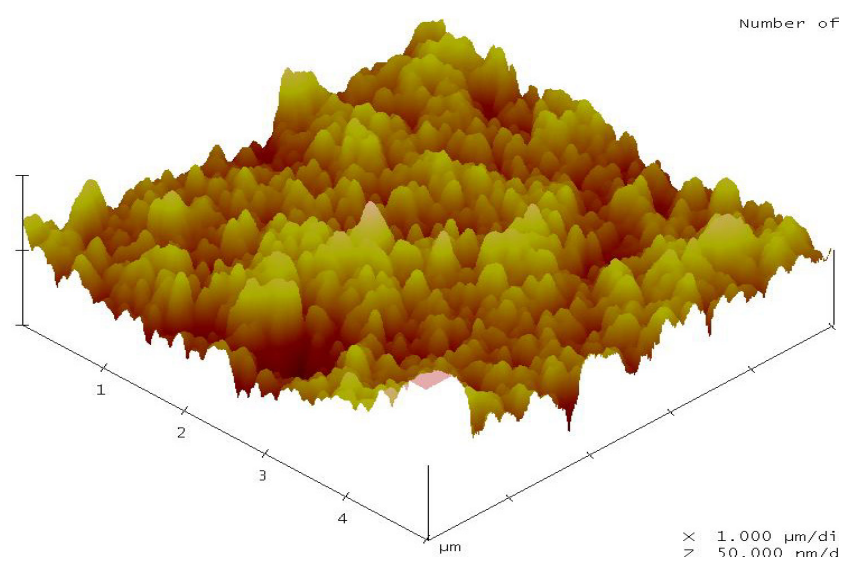

Figure 7. 3D AFM image of porous structure

The attenuation of the reflectivity is due to scattering and transmission at the porous and bulk interfaces. This parameter has its significant role in order to enhance the light conversion efficiency of solar cells. In order to confirm the 
applicability of porous silicon to be used as antireflection coating, the reflection measurement was done. We have characterized various samples of porous silicon and it is observed current density or etching time is a key factor for the variation in refractive index or porosity of the layer which results deviation in reflectivity. The observed reflectivity is found to be in between $\sim 11-12 \%$ in the range of $400-800 \mathrm{~nm}$ which is shown in figure 8 .

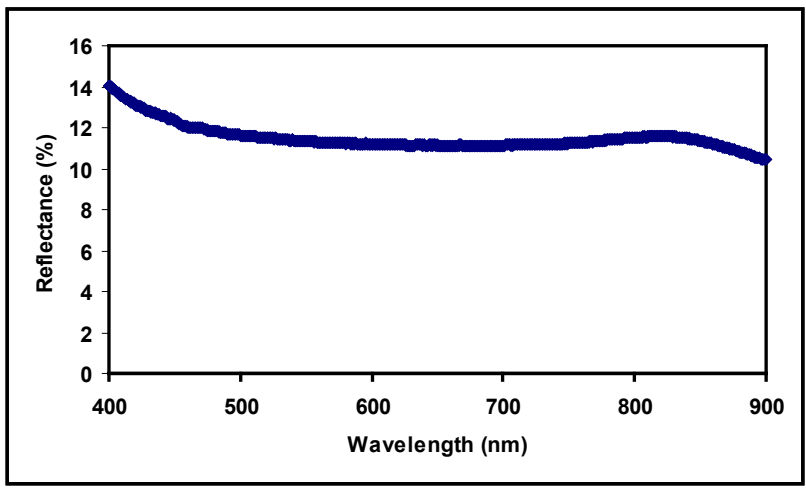

Figure 8. Reflectivity of porous structure as a function of wavelength.

As the current density varies the refractive index is changed which causes variation in the porosity of the layer as a result, the reflectance is varied.

\section{Conclusion}

The porous silicon layers were characterized and studied to observe as minimum as possible reflection. This low reflection porous silicon layer is useful as antireflection coating of solar cells. The ellipsometry measurements showed that as the etching time increases the thickness and refractive index of porous silicon layer increases and decreases respectively. The XRD spectra of porous silicon layer showed a sharp peak at $2 \theta=69^{\circ}$, which confirmed that the crystalline properties of porous silicon was not disturbed after electrochemical etching process. The surface hydrides on the surface of porous silicon were responsible for the 'red' band photoluminescence and it has been attributed to $\mathrm{SiHx}$ groups as confirmed by FTIR spectra. A broad peak of red emission was also confirmed by photoluminescence measurement. AFM measurement has confirmed the pyramid like surface over the entire surface which is responsible for the reduction of reflection of photons.

\section{REFERENCES}

[1] L. T. Canham, Appl. Phys. Lett., Vol. 57, 1046 (1990).

[2] Asmiet Ramizya, Z. Hassana, Khalid Omara, Y. Al-Dourib and M.A. Mahdi, Appl. Sur.Sci., Vol. 257, 6112 (2011).

[3] Khaldun A. Salman , Z. Hassan and Khalid Omar, Int. J. Electrochem. Sci., Vol.7, 376 (2012).

[4] S. Setzu, P. Ferrand, R. Romestain, Mater. Sci. \& Engg., Vol. 70, No. 34 (2000).

[5] P. Ferrand, D. Loi, R. Romestain, Appl. Phys. Lett., Vol. 79, No. 19, 3017 (2001).

[6] J. E. Lugo, H. A. Lopez, S. Chan, P. M. Fauchet, J. Appl. Phys., Vol. 91, No. 8, 4966 (2002).

[7] C. J. Oton, L. Dal Negro, Z. Gaburro, L. Pavesi, A. Lagendijk, and D. S. Wiersma, Phys. Stat., Sol. Vol. 197, No. 1, 298 (2003).

[8] V. Agrawal, J. A. del Rio, Appl. Phys. Lett., Vol. 82, No. 10, $1512(2003)$

[9] Huimin Ouyang, Christopher C Striemer and Philippe M Fauchet, Appl. Phys. Lett., Vol. 88, 163108-1 (2006).

[10] Dominici and Francessco michelotti, Appl. Phys. Lett., Vol. 91, 241109 (2007). 